\title{
Sensorless Iterative Solar Tracking In Multiple On-Grid Photovoltaic Generators With Improved Tracking Strategy
}

\author{
Cittolin, Guilherme F.; Mazuroski, Jéssica V.; Gonçalves, Rafael C.; Kroetz, Marcel G.; Pece, Carlo A. Z.; \\ Santos, Winderson E. \\ Laboratório de Processamento Eletrônico de Energia, Departamento Acadêmico de Eletrotécnica \\ Universidade Tecnológica Federal do Paraná - UTFPR \\ Campus Curitiba - Avenida Sete de Setembro, 3165 - Curitiba/PR, Brasil - CEP 80230-901 \\ Phone: +55 41 3310-4778, e-mail: gfcittolin@gmail.com, jevmaz@gmail.com, ralfcollar@gmail.com, \\ marcelkroetz@utfpr.edu.br, carlopece@utfpr.edu.br,winderson@utfpr.edu.br.
}

\begin{abstract}
The efficiency of the energy conversion in photovoltaic panels varies according to the angle of light incidence. A new method of iterative repositioning of the panels at maximum-efficiency direction has been proposed. This method frees up the use of sun position sensors and uses the instantaneous power delivered to the power line by each panel, among a series of photovoltaic panels, as a feedback in determination of the best position in a closed-loop operation. The proposed repositioning method, as well as the repositioning dynamic of a series of photovoltaic panels, has been modeled. The system has been simulated in a single parallel-rays light source scenario. In simulations, the convergence of panels to the best energy conversion position was observed.
\end{abstract}

\section{Key words}

Solar Tracker, Solar Energy, Photovoltaic Panels, Sensorless Control, Iterative Control.

\section{Introduction}

The increasing demand for electric power and the emerging need of reduction of the human activities impact on the environment has motivated much progress on the development of alternative energy sources [1], with special attention to renewable energy sources, said sustainable [2]. In this context, stands the study of the luminous energy to electrical energy conversion [3]. This one is shown to be gainful considering the reduced environmental impact, in addition to its high reliability and low cost of maintenance and operation [4], [5].

Nevertheless, the use of photovoltaic (PV) panels still exhibits a slow and progressive growth, mainly because its elevated cost per watt(U\$/W) [6]. Thus, it is noted the necessity of improving the efficiency of PV panels, with the purpose of minimizing its implementation cost.

The critical point observed on optimizing the use of PV panels consists mainly on the control of the orientation direct through the light source direction [7] and the control of his load operation [8], [9], in different situations of irradiation and power demand.

The active methods for panel reorientation through the light source direction latterly used can be sorted in two main groups: the position control using sensors of the light source position; and the geoastronomical based. The first, also known as Solar Tracker, basically consists in luminosity sensors connected to a controller that acts on the correction of the position of the panel in relation to the sun's position [4]. On the other hand, the geoastronomical control is based on a reference table of the sun's position through the year and on the open loop orientation of the photovoltaic panels by the previously calculated best position. [2], [3], [10]. In both cases, the main function of the position control is to ensure that the sun light incidence is the most perpendicular to the PV panels as possible, operating in one or two rotating axes (Fig. 1).

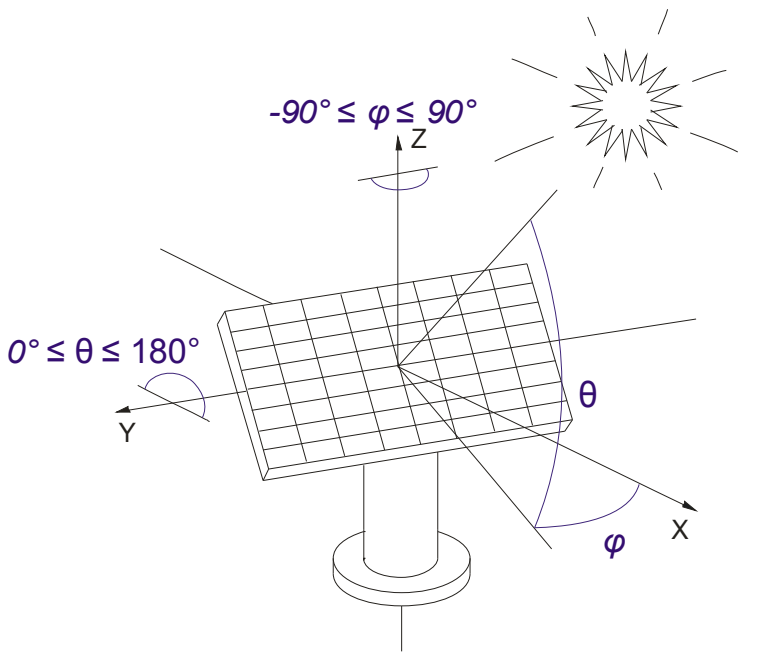

Figure 1. Orientation axis of a photovoltaic panel.

$\varphi$ - east-related orientation. $\theta$ - horizon-related elevation.

In this context, a closed loop control strategy of PV panels was looked for, without using sensors of the position of light source. Therefore, it was assumed that the instantaneous power generated by each PV panel, of a set of panels, can be used as an indicator of its relative orientation to the light source [11]. Thus, it enables the sensorless reorientation of the PV panels, even in a challenging scenario in which all the PV panels are connected to the power line through individual DC/AC inverters. 


\section{Sensorless Solar Tracking Approach}

The main goal of this work was to enhance the sensorless control strategy, as presented by [11], to orientate a set of PV panels to the direction of light source, without using sun position sensors, thereby, considering also the following additional restrictions:

1) the control must be given in closed loop

2) the instantaneous power generated by each PV panel has to be used as feedback control

3) the control must be performed in a scenario in which all generators were connected to the power line by his own DC/AC converters (Fig. 2)

4) the movement of PV panels has to be the least possible to reach the convergence point

5) no adjustment or manual configuration must be necessary during the installation and operation of the control system.

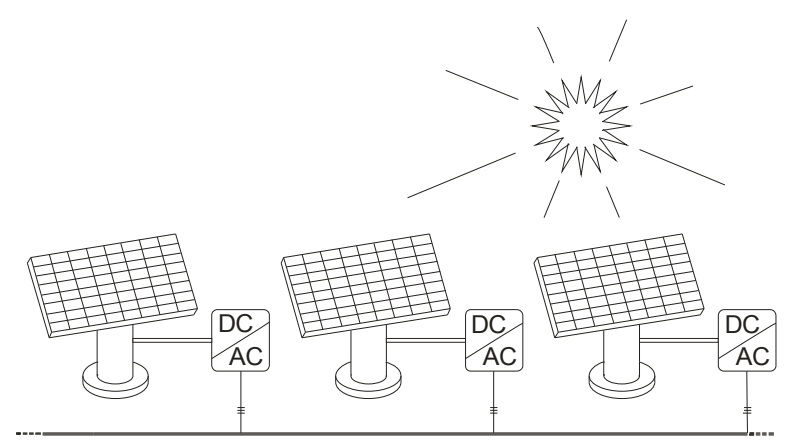

Figure 2. Photovoltaic panels connected to the power line by individual $\mathrm{DC} / \mathrm{AC}$ converters

The power delivered by a solar panel to the grid, when connected through an individual $\mathrm{CC} / \mathrm{CA}$ inverter, depends on the load of the grid, which can very dynamically. Moreover, the voltage generated by a solar panel depends on the instantaneous power delivered, while their inverter tracks the maximum power point. This lead to a situation where neither the instantaneous power nor the generated voltage cold be used as a feedback indicator during the solar tracking.

The reached solution to this feedback problem in sensorless solar tracking, as suggested by [11], is reorientates the solar panels as a whole set, considering his instantaneous power delivered to the grid relative among themselves as a feedback indicator. This way, the dynamically fluctuations of the load on the grid, that is common to all the solar panels, cold be dismissed, and the relative power delivered cold be satisfactorily used, making the sensorless tracking suitable without knowing the gradient of the sun orientation.

Beside, each solar generator could be connected independently to the line through its own DC/AC inverter, improving the system modularity and scalability, and also maximizing the system efficiency through individualized tracking of the maximum power point.

\section{Solar Panel Attitude Modeling}

It was also an aim to obtain a compact and convenient way of expressing the attitude of the PV panels using vector arithmetic, in order to unify the mathematical treatment used on the simulation and the control.

A way of representing attitude on solar generators was looked for, related to the work of [12]. The used notation is based on the representation of the attitude as threedimensional unitary vectors, and uses transformation matrices and its associated matrices algebra to model the system and perform the simulations.

In the context of this work, attitude was considered as the orientation, or angular position, of the solar panel relative to the sun or his reference, as described in figure 3, and the needed Euler rotations to perform their alignment are expresses trough rotation matrices.

A rotation matrix is a matrix that is used to perform rotation in a coordinates in Euclidean space around a Euler angle. To rotate a vector using a rotation matrix, his coordinates have to be expressed in a column vector, and the rotated coordinates are obtained multiplying this vector by the rotation matrix. Rotation matrices are a convenient way to algebraic express a rotation, and were chosen because their compactness and elegance.

The tree basic rotations, around each of the cartesian axes, as seen in figure 3 , cold be represented by rotation matrices as follow.

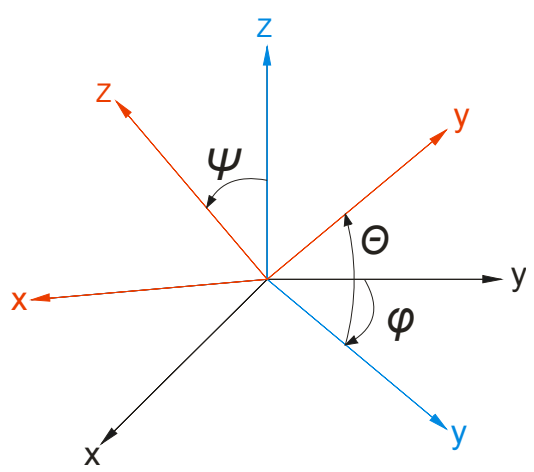

Figure 3. Euler axes and their respective rotation angles. Coordinate space fixed drawn in blue. Coordinate space rotated drawn in red.

Considering coordinate space rotated in reference to a coordinate space fixed, the equations 1 to 3 describe their respective rotation matrices, around the $\mathrm{Z}, \mathrm{Y}$ and $\mathrm{X}$ axes, respectively.

$$
\left[T_{b}^{a}\right]=\left[\begin{array}{ccc}
\cos \varphi_{a b} & -\sin \varphi_{a b} & 0 \\
\sin \varphi_{a b} & \cos \varphi_{a b} & 0 \\
0 & 0 & 1
\end{array}\right]
$$




$$
\begin{aligned}
& {\left[T_{b}^{a}\right]=\left[\begin{array}{ccc}
\cos \theta_{a b} & 0 & \sin \theta_{a b} \\
0 & 1 & 0 \\
-\sin \theta_{a b} & 0 & \cos \theta_{a b}
\end{array}\right]} \\
& {\left[T_{b}^{a}\right]=\left[\begin{array}{ccc}
1 & 0 & 1 \\
0 & \cos \psi_{a b} & -\sin \psi_{a b} \\
0 & \sin \psi_{a b} & \cos \psi_{a b}
\end{array}\right]}
\end{aligned}
$$

Where:

$a$ - means coordinate space fixed

$b$ - means coordinate space rotated

From these tree rotations matrices, only the two ones corresponding to the rotation around the $\mathrm{Z}$ and $\mathrm{Y}$ axes were used to obtain the cosine of the light incidence angle, because of this cosine is insensitive among $\mathrm{X}$ axis rotations. But two compound rotation matrix was necessary to obtain the final sun to panel rotation matrix. For following equations, "p" is used as solar panel and "s" as sun. These equations describe the necessary rotations from a common reference to sun and to panel, and then the final compound rotation direct from the sun to the panel.

The first rotation is around the $\mathrm{Z}$ axis, seen in equation 4 . With help of an auxiliary coordinate space, named "a", as the coordinate space rotated; the rotation angle $\varphi$ defines the rotations around the $\mathrm{Z}$ axis in reference to the reference coordinate space, named " $r$ ", as the coordinate space fixed.

$$
\left[T_{a}^{r}\right]=\left[\begin{array}{ccc}
\cos \varphi_{s} & -\sin \varphi_{s} & 0 \\
\sin \varphi_{s} & \cos \varphi_{s} & 0 \\
0 & 0 & 1
\end{array}\right]
$$

\section{Where:}

$r$ - means reference coordinate space

$a$ - means auxiliary coordinate space

$\varphi_{s}-$ means rotation angle around $\mathrm{Z}$ axis

The second rotation is around $\mathrm{Y}$ axis, seen in equation 5 . Now, with "a" as the coordinate space fixed, $\theta$ defines the rotations around the $\mathrm{Y}$ axis in reference to the sun's coordinate space, as the coordinate space rotated.

$$
\left[T_{s}^{a}\right]=\left[\begin{array}{ccc}
\cos \theta_{s} & 0 & \sin \theta_{s} \\
0 & 1 & 0 \\
-\sin \theta_{s} & 0 & \cos \theta_{s}
\end{array}\right]
$$

\section{Where:}

$a$ - means auxiliary coordinate space

$s$ - means sun coordinate space

$\theta_{s}-$ means rotation angle around $\mathrm{Y}$ axis
Then, to obtain the compound rotation matrix from " $r$ " to "s", is necessary to get the matrix multiplication of the two previews rotation. As seen in equation 6 .

$$
\left[T_{s}^{r}\right]=\left[T_{a}^{r}\right]\left[T_{s}^{a}\right]
$$

Where:

$r$ - means reference coordinate space

$s$ - means sun coordinate space

$a$ - means auxiliary coordinate space

In the same way, the compound rotation matrix from " $p$ " to " $r$ " was obtained, as seen in equation 7. But with " $p$ " as the coordinate space rotated in equations 4 and 5 and 6.

$$
\left[T_{p}^{r}\right]=\left[T_{b}^{r}\right]\left[T_{p}^{b}\right]
$$

Where:

$r$ - means reference coordinate space

$p$ - means panel coordinate space

$b$ - means another auxiliary coordinate space

The final rotation matrix is composed by the inverse rotation from " $p$ " to " $r$ " then from " $r$ " to " $s$ ". The inverse rotations from " $p$ " to " $r$ " was obtained as the transposed rotation matrix from " $r$ " to " $p$ ", as seen in equation 8 .

$$
\left[T_{p}^{r}\right]^{T}=\left[T_{p}^{b}\right]^{T}\left[T_{b}^{r}\right]^{T}=\left[T_{b}^{p}\right]\left[T_{r}^{b}\right]=\left[T_{r}^{p}\right]
$$

Where:

$r$ - means reference coordinate space

$p$ - means panel coordinate space

$b$ - means another auxiliary coordinate space

Finally, the rotation matrix from "s" to "p" was obtained by the matrix multiplication of this two rotation matrix, resulting in the final direct rotation matrix, as seen in equation 9 .

$$
\left[T_{s}^{p}\right]=\left[T_{r}^{p}\right]\left[T_{s}^{r}\right]
$$

\section{Where:}

$p$ - means panel coordinate space

$r$ - means reference coordinate space

$s-$ means sun coordinate space

In this final rotation matrix, the first member of the first column correspond to the cosine of the angle between the solar panel's normal and the sun light incidence angle, named $\gamma$, as seen in equation 10 .

$$
\cos (\gamma)=\sin \left(\theta_{p}\right) \sin \left(\varphi_{p}\right)+\cos \left(\theta_{s}\right) \cos \left(\theta_{p}\right) \cos \left(\varphi_{p}-\varphi_{s}\right) \text { eq. } 10
$$


Were:

$\gamma$ - means angle between solar panel's normal and sun light ray

$\theta_{\mathrm{s}}-$ means rotation angle from sun to reference around the $\mathrm{Y}$ axis

$\theta_{\mathrm{p}}$ - rotation angle from panel to reference around the $\mathrm{Y}$ axis

$\varphi_{s}$ - rotation angle from sun to reference around the $\mathrm{Z}$ axis

$\varphi_{p}$ - rotation angle from panel to reference around the $\mathrm{Z}$ axis

\section{Sensorless Solar Tracking Algorithm}

To accomplish the proposed objectives, a improved tracking strategy was formulated and implemented based on an extended local unimodal sampling optimization algorithm.

As described in [13], a local unimodal sampling allow the optimization of a function without the knowledge of its gradient, and basically consist of randomly pick up samples of the search space and keep one of these samples as a candidate solution, only if it perform better than the previews one.

A extended version of that algorithm was applied as solar tracking algorithm, using the power delivered by a solar panel to the grid relative among each other panels, in a set of panels, as feedback to optimize the entire set orientation. Additionally, only the less performing panel is reoriented, keeping the maximum possible power generating during the solar tracking and assuring the minimum movement of solar panels. This algorithm could be described by the following steps:

1. Initialize the solar panels orientations randomly among possible orientations;

2. Pick the less performing solar panel, comparing his instantaneous power delivered to the grid with all the others panels in the same set;

3. Reorientate this solar panel in direction of the best performing solar panel, adding a random disturbance in his final orientation;

4. Reduce the random disturbance and resume in the second step, until the desired number of iterations was reached or a minimum diversity in panel's orientations was obtained.

5. Wait for a predefined delay and restart in second step;

The random disturbance is needed to maintain a minimum diversity in the solar panels orientations, allowing the convergence of the set to the best orientation, and should be always expressive. As pointed by [13], better results are obtained starting with a greater disturbance and reducing this disturbance gradually among the iteration steps.

Finally, the delay and posterior restart after the convergence is necessary because the dynamic nature of the best orientation, allowing the tracking behavior of the solar panels set all day long.

\section{Simulation Results}

The proposed algorithm was simulated considering a set of eight solar panels, equipped with solar tracking actuators of two degrees of freedom, as illustrated by the figure 1. For this simulation, the sun was considered oriented at $10^{\circ}$ from east to north and $60^{\circ}$ of elevation from the horizon.

For the purpose of this simulation, the performance of each panel was considered as the cosine of the angle between the solar panel normal and the light incidence, by the fact of the relative efficiency of a solar panel is strongly dependent on the light incidence angle. This cosine was obtained with the equation 10 , as explained previously.

The solar panels were initialized in random orientations, limiting their movement by $-\Pi$ to $\Pi$ in rotation and 0 to $\Pi$ in elevation, as shown in figure 4.

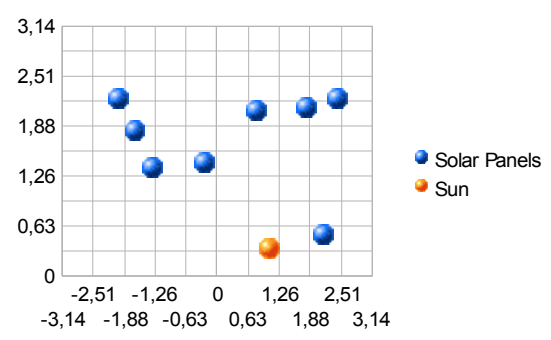

Figure 4. Initial solar panels orientations

In each iteration, the less performing solar panel was picked and reoriented in direction of the best performing solar panel, adding to his orientation a random disturbance of $\pm 0.5 \Pi$ on rotation and $\pm 0.125 \Pi$ on elevation at $1^{\text {st }}$ iteration; decaying exponentially to $\pm 0.0005 \Pi$ and $\pm 0.000125 \Pi$, respectively, at the $50^{\text {th }}$ iteration.

The figure 5 shows the relative performance of each solar panel during the simulated iterations. In this figure, we could observe a satisfactory convergence of the panels near the $30^{\text {th }}$ iteration.

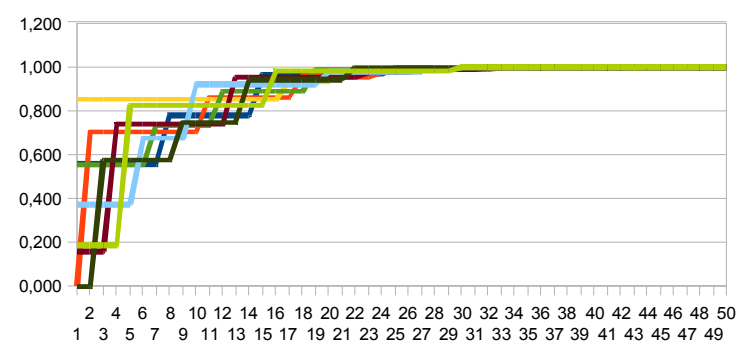

Figure 5. Relative performance of each panel during the simulated iterations

This convergence can be observed too in the figures 6 and 7. The figure 6 shows the solar panels orientations 
after 10 iterations and the figure 7 shows their orientations after twelve iterations.

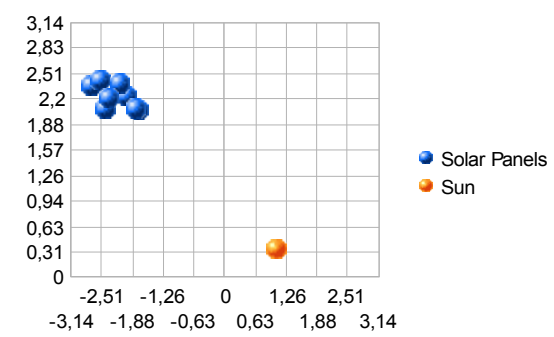

Figure 6. Solar panels orientation after ten iterations

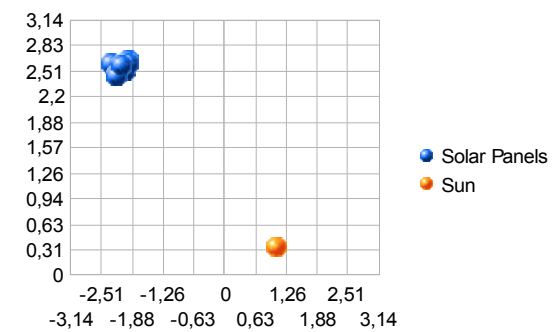

Figure 7. Solar panels orientations after twelve iterations

Is interesting explain at this moment who the solar panels apparently converge to a distinct orientation other than direct to the sun. This occurs because with the simulated freedom of the panels, they could archive the same orientation by two distinct coordinates that overlap. This way, the two distinct point in the map are actually the same orientation described by two distinct coordinates.

The figure 8 shows the surface of relative performance through this overlapping coordinate space, considering the sun at $\varphi=10^{\circ}, \theta=60^{\circ}$. In this surface, we could observe two maximums, besides the unimodality of the plotted function.

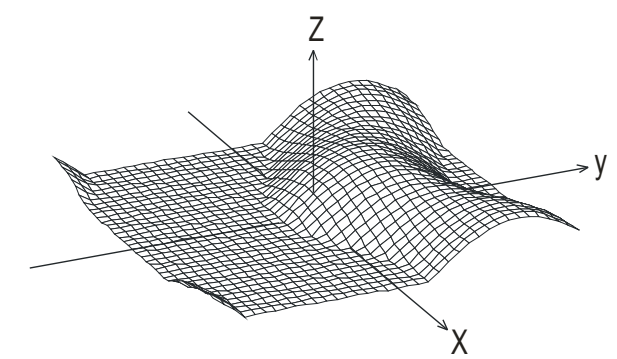

Figure 8. Surface of the performance of solar panels orientations relative to the sun. Sun at $\varphi=10^{\circ}, \theta=60^{\circ}$.

\section{Conclusions}

The proposed sensorless solar tracking approach and algorithm has been simulated and the simulations results show a quick convergence in just a few iterations. The final implemented strategy agrees with the imposed restrictions and seems to be ready for concrete use.

The absence of sensors, configurations or calibrations and the observed results, lead to the following implied advantages over sensored and georeferenced solar trackers:

1) lower cost, dispensing solar position sensor, its calibrations and maintenance;

2) ease of installation, dispensing georeferencial coordinates determination and permanent calendar adjustments and maintenance;

3) on-grid operation and full compatibility with one $\mathrm{DC} / \mathrm{AC}$ converter per solar generator.

However, the way of operation of this sensorless approach need at least two solar generators, and all the solar panels in the same set need to be homogeneous.

The results of this work are being validated in a concrete under-construction PV panel array, composed of three experimental solar generators equipped with 2-axis solar tracker actuators each one.

Furthermore, a self-learning approach, combined with sensorless tracking, is under study, inspired by [6], aiming at minimizing installation misalignment and yet less power consume in solar tracking.

\section{References}

[1] Sharaf, A. M. Yang, L. 2005. An Efficient Photovoltaic DC Village Electricity Scheme Using a Sliding Mode Controller. IEEE - Conference on Control Applications. Canada. 2005.

[2] Chun-Sheng, W. Yi-Bo, W. Si-Yang, L. Yan-Chang, P. Hong-Hua, X. 2008. Study on Automatic Sun-tracking Technology in PV Generation. Electric Utility Deregulation and Restructuring and Power Technologies. China. 2008.

[3] Stalter O., Burger B. Integrated Solar Tracker Positioning Unit in Distributed Grid-Feeding Inverters for CPV Power Plants. IEEE - Conference on Industrial Technology, ICIT. 2009.

[4] Han, D. Wang, Zc. Shen, H. Xu, G. Li, F. 2009. Research and Design on a Sun-tracker. Sustainable Power Generation and Supply. 2009.

[5] Wai, R. Wang, W. Lin, C. 2008. High-Performance StandAlone Photovoltaic Generation System. IEEE Transactions on Industrial Electronics. Vol. 55, No.1, Jan. 2008.

[6] Anton, I., Perez F., Luque I. Sala G. 2002. Interaction between Sun tracking deviations and inverter MPP strategy in concentrators connected to grid. Proceedings of IEEE Photovoltaic Speccial Conference, 2002.

[7] Serhan, M. El-Chaar, L. Two Axes Sun tracking System: Comparision with a Fixed System. International Conference on Renewable Energies and Power Quality - ICREPQ'10. 2010 .

[8] MacIsaac, L., Knox., A. Improved Maximum Power Point Tracking Algorithm for Photovoltaic Systems. International Conference on Renewable Energies and Power Quality ICREPQ'10. 2010. 
[9] Pellegrin, Juliano. P. Gules, Roger. 2009. Otimização da Energia Gerada em Painéis Fotovoltaicos em Sistemas isolados da Rede Elétrica. I Mostra Anual de Pesquisa e Pós-Graduação do CPGEI. 2009.

[10] Duarte, F., Gaspar P. D., Gonçalves, L. C. Two axis solar tracker based on solar maps, controlled by a low-power microcontroller. International Conference on Renewable Energies and Power Quality - ICREPQ’10. 2010.

[11] Gonçalves, R. C., Pimentel, C. E. F., Cittolin, G. F., Kroetz, M. G., Andrea, C. Q., Santos, W. E. Reposicionamento Sensorless Iterativo de Múltiplos Painéis Fotovoltaicos Conectados à Rede Elétrica. Congresso
Brasileiro de Automática. Bonito/MS, Brasil. 2010.[14] Anton, I., Perez, F., Luque, I.., Sala, G. Interaction between Sun tracking deviations and inverter MPP strategy in concentrators connected to grid. Photovoltaic Specialists Conference, 2002. Record of the Twenty-Ninth IEEE. 2002.

[12] Pece, C. A. Z. An Engineering Vector-Like Approach to Attitude Kinematics \& Nominal Attitude State Tracking Control. PhD Thesis. Instituto Tecnológico de Aeronáutica, São José dos Campos, SP, Brasil. 2002.

[13] Pedersen, M. E. H., Chipper, A. Local Unimodal Sampling. Hvass Laboratories Technical Report no. HL0801. 2008 